Title:

Cocaine Rituals in Club Culture: Intensifying and Controlling Alcohol Intoxication

Author:

Marit Edland-Gryt ${ }^{1,2}$

${ }^{1}$ Department of Alcohol, Tobacco, and Drugs, Norwegian Institute of Public Health

${ }^{2}$ Department of Sociology and Human Geography, University of Oslo, Norway

Corresponding author:

Edland-Gryt, Marit, Department of Alcohol, Tobacco, and Drugs, Norwegian Institute of Public Health, Post Box 222-Skøyen, 0213 Oslo, Norway. E-mail address: Marit.EdlandGryt@fhi.no. 


\title{
Title: Cocaine Rituals in Club Culture: Intensifying and Controlling Alcohol Intoxication
}

\begin{abstract}
Clubbing is an important part of the nighttime economy, and cocaine use is, for some young people, an essential part of this clubbing culture. However, the interaction rituals around the use of powder cocaine in this context remain understudied. This study is based on qualitative interviews with young adult recreational cocaine users $(n=28)$, and explores how they use cocaine in club settings, in relation to rituals and drinking culture. The analysis identified three main explanations for using cocaine: (1) unity with friends because of shared transgression, (2) the high as a "collective effervescence", and (3) the possibility to control, extend and intensify drinking to intoxication. These three explanations illustrate how cocaine rituals were deeply integrated in drinking-to-intoxication rituals, and how the illegality of cocaine use reinforced feelings of unity with friends. In the nighttime economy, cocaine use and its related rituals are used to intensify and control alcohol-fuelled partying.
\end{abstract}

\section{Keywords}

Cocaine use, interaction rituals, controlled loss of control, alcohol, recreational substance use, young adults, intoxication. 


\section{Introduction}

Clubbing is a central part of many young adults' lives. In Norway, the club culture is alcohol driven and drinking to intoxication is a common phenomenon in the Nordic countries (Kuntsche, Rehm, \& Gmel, 2004; Mäkelä et al., 2001), as in other parts of the Western world (Measham \& Brain, 2005; Moore, 2010). Alcohol use encompasses certain rituals, and drinking together carries important symbolic and social meanings (Douglas, 2013; Järvinen, 2003), such as creating a shared feeling of unity (Rothenbuhler, 1998). Despite the cultural primacy of drinking, many also wish to augment their drinking experiences and to make the night out more special by using other substances, such as cocaine. This study aims to advance our understanding of these phenomena, by focusing on the users' perceptions of their cocaine use in relation to drinking to intoxication and drinking rituals. In addition, it also aims to show how powder cocaine use may be an integral part of the alcohol-based club culture, and how it intensifies drinking to intoxication in club settings. This study challenges the dominant view of cocaine use as primarily pharmacologically driven (i.e., for "the high" and euphoria), and facilitates an understanding of cocaine use from a more complex sociocultural perspective.

Currently, cocaine is ranked as the second most frequently used illegal drug in European countries (EMCDDA, 2019), and appears to be repositioning itself as the stimulant drug of choice. Cocaine is often described as a problematic drug, due to its associations with dependence (Dackis \& O'Brien, 2001; Nutt, King, \& Phillips, 2010; Uosukainen, Tacke, \& Winstock, 2015). In the Norwegian nightlife context, powder cocaine is the most frequently used illegal drug after cannabis, while there is little use of crack cocaine (Nordfjærn, Bretteville-Jensen, et al., 2016) and, in a study of the Norwegian student population, cocaine use ranked third, after cannabis and MDMA (Heradstveit et al., 2020). Powder cocaine is seen as a drug that is frequently used by socio-economically privileged groups: "The glamour makes the difference", notes a Danish study (Demant, Ravn, \& Kaae, 2011). Unlike cannabis, which is much more widely accepted, and heroin, which is not accepted at all in clubs, cocaine occupies an ambiguous position, being perceived as neither clearly harmless nor clearly dangerous (Demant \& Ravn, 2010). 
Previous research on the use of cocaine has been dominated by pharmacological and toxicological approaches (Esteve-Arenys et al., 2017; Kuypers, Steenbergen, Theunissen, Toennes, \& Ramaekers, 2015; Zyoud et al., 2017). Studies have shown that the combination of cocaine and alcohol produces a new substance in the liver, cocaethylene (Harris, Everhart, Mendelson, \& Jones, 2003), which is a pharmacologically active metabolite of cocaine formed in the body (Jones, 2019). This metabolite takes longer to be eliminated than cocaine alone ${ }^{1}$, may in itself increase euphoria, and may be one of the reasons why the combination of cocaine and alcohol is a favored combination (Andrews, 1997). Psychological aspects of cocaine addiction have also been thoroughly studied (see, for example, Gawin, 1991). Only a handful of studies have examined the cultural aspects of cocaine use (Decorte \& Slock, 2005; Grinspoon \& Bakalar, 1985; Waldorf, Reinarman, \& Murphy, 1992; Williams, 1989), typically investigating the use of both powder and crack cocaine, or mainly crack cocaine (Fagan, 1994), despite potentially substantive differences between these two user groups (Martin, Macdonald, Pakula, \& Roth, 2014; Palamar, Davies, Ompad, Cleland, \& Weitzman, 2015). In the current study, cocaine refers to recreational use of powder cocaine, which has not been much studied within social science. Decorte (2001), drawing on data from Belgium, examined both snorting and injecting use and user perceptions, finding that becoming a "controlled cocaine user" was primarily a process of gaining knowledge about the product, and that the social definition of drugs and their users were important. One mixed-method study from the Netherlands examined recreational cocaine use (van der Poel, Rodenburg, Dijkstra, Stoele, \& van de Mheen, 2009), and found that cocaine was used together with alcohol on the same occasion, but provided limited insights into the culture surrounding use. In a study from Denmark, Demant (2010) investigated combined alcohol and cocaine use in a club setting. He found that some used cocaine to control their alcohol consumption, and as a way to manage a night at a club, and that cocaine was perceived as a social drug.

The current study builds on these findings and extends them by further investigating explanations for using cocaine in combination with alcohol from a theoretical perspective, applying interaction ritual theory (Collins, 2004). In this study, it is the motivation for using cocaine, and not the negative aspects of cocaine use, that is primarily in focus. 


\section{Interaction rituals related to drinking and clubbing}

"Society is held together more intensely at some moments than at others. And the "society" that is held together is no abstract unity of a social system, but is just those groups of people assembled in particular places who feel solidarity with each other through the effects of ritual participation and ritually charged symbolism". (Collins, 2004, p.41)

Theoretical frameworks of interaction rituals related to intoxication and the context around substance use can help us understand more about how cocaine users perceive and comprehend their use of the drug within drinking culture. For alcohol use, various terms are used to describe drinking with the aim of intoxication, for instance "binge drinking" and "heavy drinking" (Berridge, Herring, \& Thom, 2009; Gmel, Rehm, \& Kuntsche, 2003). In this study, “drinking to intoxication” will be employed when referring to the participants' drinking while clubbing. While drinking occasions may occur less frequently in the Nordic countries than in Southern Europe, they are more likely to result in alcohol intoxication (Babor et al., 2003; Rolando, Törrönen, \& Beccaria, 2014). Drinking to intoxication and cocaine use are both embedded in interaction rituals, and this will be further explored in the following.

Rituals consist of specific actions, which might be regarded as types of ceremonies. These rituals permeate every aspect of our social life (Goffman, 2008 [1967]), and are just as necessary to our social life as food is to the nurture of our physical life (Durkheim, 1965 [1915]). Rituals also shape drug-use behaviors, since drug use often takes place in social groups and through repeated procedures. Comprehending these rituals can contribute to a broader understanding of drug use in the nightlife setting. This study is rooted in a tradition based on Durkheim (1965 [1915]) and Goffman (2008 [1967]) of how to comprehend rituals and social situations, as was true of earlier studies (Järvinen, 2003; Rothenbuhler, 1998). Järvinen (2003) illuminated how alcoholism is something "eminently social", by revealing the collective structures where people accept the command of the ritual as if it were a material power they could not control. Douglas underlined the importance of the social aspects of alcohol use: "drinking is essentially a social act, performed in a recognized social context" (Douglas, 2013, p. 4), and thereby acknowledges that the setting is essential and that alcohol use is fundamentally social in nature. Cocaine use will be analyzed in accordance with Douglas' framework of alcohol drinking, and with Goffman's idea of "micro-rituals", "ritualized behavior" (Goffman, 2008 [1967]) and the importance of euphoria in encounters (Goffman, 1990), while linking it to clubbing. 
Important aspects of our social lives are driven by what Collins terms interaction ritual chains (Collins, 2004), where successful rituals create group membership and invigorate individuals with emotional energy (Collins, 1993). Rituals concern mutually focused emotion and attention that produce a shared reality, which, in turn, generates solidarity and symbols of group membership. Emotional energy is an empirically based concept, and explained as a product of interaction rituals (Collins, 2004); it can be seen as a kind of metaphorical "electricity" that is created through shared rituals in special situations (2004, p.39). The group membership can also create solidarity and, when applied to the drug-use settings, certain aspects of interaction ritual chains can help understand the relationship between emotional energy and the settings in which drugs are used, and how rituals can endow symbolic objects with significance (Collins, 2004, p. 38). It is through rituals that groups create their symbols (Collins, 2004, p.26), and the situation where cocaine is shared between friends is analyzed as an example of a situation with emotional energy. The point of departure for Collins' theory is situations rather than individuals, and this theory of situations concerns momentary encounters between humans charged up with emotions and consciousness, including as a result of previous chains of encounters (Collins, 2004, p.3).

Collins' framework will be employed to help understand various ritualistic aspects of cocaine use among young adults, in "collective effervescence" (Collins, 2004, p.105), especially as concerns how participants perceive their feelings of unity with friends. This collective effervescence is a strong, shared emotional experience produced by collective rituals (Collins, 2004; Tutenges, 2013). The value of these rituals can be elucidated by the concept of "sneaky thrills", as explained by Katz (1988), which can also create emotional energy, social bonds and solidarity for those involved (Kavanaugh \& Anderson, 2008). Katz (1988) explains "sneaky thrills" as a product of crimes that share an appeal to young people in particular, regardless of material gain. In this study, the framework of rituals and collective effervescence in the drinking culture will be expanded to show how cocaine fits into these rituals by analyzing what young adults recount about situations and settings where cocaine is used.

While substance use is common within the nightlife context, it appears to be characterized by the young adults' seemingly contradictory desires to be both intoxicated and in control (Measham, 2002). The desired state of intoxication is to lose control, but not entirely. The concept of controlled 
loss of control highlights how young people negotiate control and loss of control when using drugs while partying, and where they maintain control in situations on the brink of chaos (Hayward, 2002; Measham, 2002). By embracing the loss of control to some extent, the young adults value risk-taking in a somewhat controlled form. Thus, the framework of controlled loss of control will help understand how cocaine is used as a means to control and extend drinking to intoxication. In addition, this study aims to extend the way in which the term "controlled loss of control" is understood - by also including an additional time aspect of how, in the user's perception, cocaine use repeatedly allows the regaining of control after prolonged drinking.

This study examines recreational cocaine use from the perspective of interaction rituals, to gain a deeper understanding of the nature of cocaine use in a nightlife setting. It is important to understand how the use of different substances is driven by the culture and rituals surrounding such use. The aim is to extend the knowledge of young adults' cocaine use within club culture contexts, by examining it as an extension of drinking to intoxication, thus providing a broader understanding of recreational cocaine use not obtainable from traditional epidemiological and pharmacological research.

\section{Materials and methods}

This study is based on qualitative interviews with 28 young recreational drug users who reported having used cocaine. Most of them were frequent users and reported that they often used cocaine while drinking at clubs. 18 of them were males and 10 were females, and they were between 20 and 34 years of age (mean age 26.7). The majority were university students or in employment and had used cocaine and other substances to various degrees when clubbing. Those in employment had a variety of occupations ranging from chef, schoolteacher and engineer, to bartender and journalist. Three were unemployed at the time of the interview. Most participants were Norwegian, four were Swedish and three were from beyond Scandinavia, reflecting the international environment of young adults in Oslo. One respondent was interviewed twice ${ }^{2}$. These 29 interviews were part of 36 in-depth interviews with participants in The Oslo Nightlife Study, a multi-method investigation of drug use in the club scene in Norway (Nordfjærn, Edland-Gryr, et al., 2016). The 28 participants selected for this particular study 
were those who reported cocaine use, the remaining participants having reported use of other drugs. The study was modeled on similar studies elsewhere, especially concerning design and recruitment (Demant, Ravn, \& Thorsen, 2010; Measham, Aldridge, \& Parker, 2001), and adjusted to the Norwegian nightlife context.

Participants were recruited outside 12 clubs and bars in the Oslo city center between 11:00 PM and 4:00 AM on weekends. The quantitative part of the study included a short survey. Those who reported use of illicit substances during the previous 12 months were invited to take part in subsequent qualitative interviews and to provide their phone number. The author contacted potential participants by phone and conducted interviews shortly afterwards ${ }^{3}$. This recruitment method has been used in other club studies (Järvinen \& Ravn, 2011; Ravn, 2012). Such an approach is sometimes described as “targeted sampling” (Watters \& Biernacki, 1989), and is evaluated as effective for studying hidden populations.

The 29 interviews were done face-to-face, and the majority lasted between 1.5 and 2.5 hours. The interview guide was pilot-tested several times. Interviews were semi-structured but open-ended, and the participants were invited to share their knowledge and experiences on their own terms (Holstein \& Gubrium, 2004). This informal approach allowed the participants to shape the interviews, and to bring up various topics. In other words, interviews mainly resembled a dialogue between interviewee and interviewer around the topic of the interview. There was no established relationship between the participants and the researcher prior to study commencement, and the participants were told about the aim of the study and the researcher's interest in the topic of the interview.

The semi-structured interview guide was used as a checklist to ensure that important topics were covered, such as the use of legal and illegal substances and the culture surrounding clubbing. Cocaine users were asked why and how they used the drug, and to describe their most recent experiences. In order to make the participants feel comfortable, they were offered a choice of interview locations, which most often ended up being the researcher's office ${ }^{4}$. The interviews were conducted by the author, a woman slightly older than the participants. Although there was no established relationship prior to the interviews, the interviewer developed a good rapport with nearly 
all participants, and this facilitated good conversations on sensitive topics. The interviewer was experienced in interviewing people who use drugs.

All interviews were audiotaped and transcribed verbatim. The interviews were also documented in field notes right after they took place, and elaborated upon and written up through the following day. The general standards for qualitative data analysis were followed (Kvale, 2009; Silverman, 2009), and content analysis was conducted (Hsieh \& Shannon, 2005). Data were analyzed thematically, placing the users' views on their own cocaine use at the center of the analysis. In addition to the predefined topics, additional topics that emerged from detailed interview readings were included in the analyses. HyperRESEARCH software was used in the coding process, and a broad range of codes - including cocaine and rituals - were created to systematize the data. The coded material included longer sections of text to provide context for the quotes. Quality checking was performed by having two researchers code two interviews: this procedure ensured that the topics were coded in the same way. The next analytical step was to reanalyze all the quotes in the relevant codes ("cocaine", "setting", "rituals", "the drug experience", and other relevant codes), and to try to identify patterns. This procedure identified common themes and led to their classification in terms of the three main explanations for cocaine use - these are presented as the main categories in the results. There is consistency between the data and the presented findings, and data saturation was discussed within the research team. Quotations are translated from Norwegian.

The study was approved by the Regional Committee for Medical and Health Research Ethics (application No. 2014/192). Participation was voluntary, following the written informed consent procedure. The participants' names and other sensitive and potentially identifying information were changed when anonymizing data.

\section{Results}

The young adults in this study described using cocaine either frequently or occasionally over several years, and had also used other illegal substances. They had all snorted powder cocaine, and none of them had tried crack. The participants typically perceived their cocaine use as having few negative 
consequences for their everyday life. They presented their cocaine use as ritualized, in that it concerned repeated actions performed in a social setting, in which emotional energy was central. The key finding was that cocaine use and alcohol intoxication and the related rituals were intertwined, and that cocaine intensified experiences of drinking to intoxication in addition to a feeling of control and alleviating next-day hangover symptoms. Cocaine was in this way seen as a strategy for the cocaine users to enable drinking over time.

The analysis below is structured around the three main explanations offered by participants to describe their cocaine use: (1) unity with friends because of shared transgression, (2) the high as a collective effervescence, and (3) the possibility to control, extend and intensify drinking to intoxication.

\section{Unity With Friends Because of Shared Transgression}

The participants said that the use of cocaine created a specific sense of unity with friends, which was much stronger than what they experienced when they were just drinking together. This was not primarily due to the drug effect itself, but related to the illegality and transgressive nature of cocaine use, and the rituals involved in the use. Participants wanted to feel fellowship with friends, and using cocaine offered them a means to strengthen this feeling. They pointed to the interaction around the use as a ritual that gave it meaning. Rick explained:

I mean, I like to do it. While doing it with other people, one gets this bond, right [...] Right then and there, it's a nice thing to do together with others. It creates a connection [...] And then I can also recall, it's you again! We got it! (Rick, 26-year-old male, frequent user)

The connection Rick talked about was also possible while drinking to intoxication without cocaine, but participants experienced it and believed it to be stronger when they used cocaine. The connection gave young adults the feeling of a kind of fictive kinship that would not emerge when drinking 
together. It gave them a sense of strong social bonds and solidarity (Kavanaugh \& Anderson, 2008), and increased emotional energy (Collins, 1993). In that sense, it can be understood in terms of an extended social "game", where the use of cocaine extended the drinking to intoxication. This was explained by the participants as something that is analyzed as a game or a social experiment, where the cocaine use produced feelings of norm violations and transgression. These feelings worked as a solid marker of friendships and strengthened social bonds through interaction rituals, for instance through snorting cocaine together with friends in clubs where this could lead to eviction. Another favored ritual was to line up the lines of cocaine in preparation for snorting. However, the strengthened sense of fellowship, through sharing this feeling of danger, created positive emotional energy among the young adults. This resonates with the special emotional energy that can be achieved by engaging in what Collins (2004) describes as "successful rituals", provided through emotional strength. Such rituals may create group membership and invigorate individuals with emotional energy. The participants recounted many different situations concerning cocaine use that evoked group membership, a feeling of fictive kinship and increased emotional energy, as Rick explained above. A successful ritual in this case could be to gather in the toilet to snort a line - because it was illegal, which made it more special. William was one of those who explained how the illegality of cocaine use contributed to the feeling:

The first time we used, it was exciting, we wanted to be tough, on the edge, because it was more of a rush to do something illegal [...] You do something illegal, but you do it together, so it is exciting. You feel a bit naughty. (William, 28-year-old male, frequent user)

The use of cocaine was seen as voluntary risk-taking that was not extreme, but quite close to the socially accepted behaviors of people William knew, although still an illegal thrill. The crossing of boundaries from licit to illicit behaviors was described as an important ritual for some participants, and the thrill was connected to this transgression. These rituals contain emotional energy (Collins, 2004) 
because the participants gather in what can be analyzed as a kind of metaphorical "electricity", which gives the users the feeling of euphoria and importance. The illegality was described as giving more edge to the experience of illegal drugs for the participants and contributed to make the use of cocaine a ritual with high intensity, and to the feeling of a thrill. Katz (1988, p.3) elaborates on the "challenge of explaining the quality of deviant experience", and one suggestion for describing it, is to explain how "sneaky thrills" contribute to the meaning for the persons involved. William meant that the illegal aspect of cocaine use contributed to a special sense of unity with friends, and the way he explains it resonates with the "sneaky crimes that frequently thrill their practitioners" (Katz, 1988, p.53). They accomplished a shared ritual that gave the feeling of a thrill, accompanied by euphoria, and this strengthened the social bonds between them.

Cocaine was valued for the substance's ability to produce unique social occasions and interactions with others. The participants talked about a collective culture of combined alcohol and cocaine use and described it as an extension of the collective alcohol intoxication culture, which gave the feeling of being an insider. These narratives parallel Collins' description of how tobacco use "rituals mark boundaries of inclusion and exclusion" (Collins, 2004, p. 297) and, in this study, the participants emphasized the feelings of being an insider when using cocaine and being part of a community. The breaking of norms explained some of the "rush", even if using cocaine for some remained a rather "normal" thing to do. Rick compared snorting cocaine to both smoking cigarettes and drinking wine, and described it in terms of just another social gathering:

Cocaine is more like a social thing, it's like smoking in my group, completely accepted. It's kind of about being social, I'd compare it to a glass of wine. (Rick, 26-year-old male, frequent user)

Cocaine use was perceived as a breach of norms in some settings, while at the same time being somewhat conventional behavior in other, small social groups. Rick explained using as an everyday 
social activity for him. Several other participants also talked as if using cocaine was a natural thing to do when partying with friends, while others perceived it as more edgy behavior. For them, cocaine use had a more symbolic value - it was like putting on a special outfit, an outfit that represented illegality, intensity, and a thrill - and that made them feel more special in turn. In both cases, cocaine use was described as pro-social, it created and enhanced the feeling of closeness with friends, and it offered a slightly different drug experience than drinking alcohol without cocaine.

Many participants favored snorting cocaine from keys and attributed this specific ritual to inspiration from movies. Cocaine users noted that they often copied customs from popular culture. The ritual involving snorting cocaine from keys made its use more special while also making it easier to hide from others, e.g. those who disapproved of drug use in clubs. Snorting from a key was also perceived as more hygienic and easier than snorting from other objects, such as the sink or toilet seat. Emily described the ritual in more detail:

Yeah, best to use the key, the key is sort of fun. When we stand outside and the bouncer comes, he might catch you, (then) you take out your keys [*makes sniffing sound*] and then it's ok. Instead of standing and trying to divide it into portions. (Emily, 20-year-old female, occasional user)

In the situation Emily describes, the context of the cocaine use was central. Because of the illegality of cocaine and the public nature of clubbing, these social encounters were of a particular sort, and the way of snorting the drug was particularly important. Emily emphasized the rituals connected to cocaine use and explained it as "doing coke". According to these users, keys were their favorite tools to snort from, and such activities can be interpreted and comprehended as micro-rituals (Goffman, 2008 [1967]) that were both cool and convenient when partying. A "key" was described as a code that could be uttered aloud without being detected, like a kind of secret language for insiders. Many of the participants noted that the common nickname for cocaine - "coke" - was too well-known. "Everybody" could understand the meaning if they heard "coke", so instead they used the term "key", 
and in this way talked in public about using cocaine without attracting unwanted attention. Talking about the key can thus be comprehended as a tacit way of communicating snorting cocaine when in the club. These types of tacitly managed rituals can be effective in changing the character of social relationships (Collins, 2004) by creating a symbol of stronger group membership, and is an example of how rituals can endow symbolic objects with significance.

Participants noted a sense of unity and feelings of closeness with friends as important explanations for cocaine use, and the illegality contributed to transgression and a feeling of connectedness that was strong, and hence gave a feeling of a "sneaky thrill" (Katz, 1988). Those who saw fellowship as a central explanation for cocaine use emphasized the importance of rituals and emotional energy. The sense of unity they described was connected to partying together with friends, and to using cocaine in addition to drinking alcohol.

\section{The High as a Collective Effervescence}

One explanation for cocaine use was, as expected, the "high" obtained, and this was experienced not only individually, but especially as a collective effervescence. Some participants described it as an extraordinary feeling of happiness, although most participants were less enthusiastic and more ambivalent about the pharmacological effect of the drug. According to the literature, cocaine's ability to produce euphoria is a valued short-time effect (Waldorf et al., 1992; Weiss, Mirin, \& Bartel, 2002), and this "high" represents the vortex of the drug experience. The psychoactive effect, or high, is often presented as the main explanation for using cocaine in popular culture. From a sociological perspective, it is clear that an over-emphasis on the pharmacological effects of the drug may lead to an underestimating of the importance of set and setting, or context, for the users' experience of drug use (Zinberg, 1984). In the present study, the feeling of cocaine was described by a few as "a royal feeling", and "breathing gold". However, most of the participants ascribed these feelings to the collective experience. Some emphasized that cocaine contributed to "shining nights", and Sylvia described the drug effect in this manner: 
You feel extremely happy, and self-esteem is really high [...] My boyfriend and I become passionate when doing coke together (laughs). You can really notice the difference even if you only snort one line, and then you talk a lot more. I also get more interested in what others say, and I can tell more fun stories. (Sylvia, 24-year-old female, frequent user)

She emphasized her high and increased confidence, and said she used cocaine every time she partied with her friends. She talked a lot about her relationship with her boyfriend, which was an important explanation for why she used cocaine, because the shared experience was magical to them. They derived a lot of joy and intimacy from its use, and described the snorting of a line as a ritual they shared in intimacy and together with friends, with increased positive emotional energy (Collins, 1993), revealing a glimpse of higher intensity than could be achieved through drinking alone. Tutenges (2013) described the feeling of excess as a state of mind young people favor, that can be reached by using drugs, in a collective effervescence. This state of mind is described as a condition of heightened intersubjectivity and intense emotions, but also acts of transgression and a strong sense of communion. This resonates with how Sylvia and other participants talked about their experiences. The use of cocaine can thus be understood in the context of Collins' (2004) interaction ritual chains, where cocaine use is analyzed as a ritual that endows the symbol with significance, through shared emotional energy and the feeling of excess when using cocaine together. It provides a feeling of a collective effervescence and emotional energy, in friendships or romantic relationships, strengthened by the use of an illegal and intoxicating substance. Collins (2004) describes the collective effervescence as a momentary state, but one that also possesses the capacity to produce more prolonged effects when it becomes embodied in sentiments of group solidarity, often affected by emotional energy. Further, Collins (2004) describes with Durkheim (1965 [1915]), how the collective effervescence can be seen as a collective consciousness. This resonates with how the participants described their cocaine use, and the high experienced, as a collective or shared consciousness where the high was part of the experience.

However, even though some respondents explained that the perceived beneficial pharmacological effects of the drug were important to them, what was particularly surprising was that 
some were ambivalent as to the importance of the obtained high. Some participants said cocaine did not create much of a change and was rather ordinary. This contradicts the historical dominant narrative of the "cocaine high" (Weiss et al., 2002). Henrik compared using cocaine to having a beer:

It's like the most expensive beer you can get. Not like "Oh, now I'm going to use drugs and get high". It's more like "Ah...let's do a line”. (Henrik, 23-year-old male, frequent user)

Even though he said that using cocaine was rather ordinary, Henrik nevertheless acknowledged that using cocaine was different from drinking beer - he used the comparison to beer to explain that the effect of cocaine was not extraordinary because of a fantastic pharmacological high, since other aspects of the drug were more important. The availability of cocaine also added to the picture of cocaine as ordinary, and participants said they could buy cocaine from both street dealers and club bouncers, in addition to regular and stable suppliers. Several stated that Oslo was a "cocaine city", and that cocaine use was an ordinary "time out" for young adults in clubs. Several participants downplayed the significance of the high, and Henrik was one of them. Many participants described cocaine as a drug that, for them, was pharmacologically of limited significance in the party culture, compared to other drugs, such as MDMA (Edland-Gryt, Sandberg \& Pedersen, 2017).

The "high" was described by some participants as an important explanation for using cocaine in club settings, while others disagreed. For those who talked about the high, it was mainly a feeling in relation to friends or lovers, and the interaction rituals related to being together, experiencing a collective euphoria and collective effervescence. The relative importance of the high is not surprising, as reflected both in movies and in historical accounts (Weiss et al., 2002). However, the results in this study depart from the typical focus in popular culture on cocaine use as driven by the special feeling of the pharmacological "high". The high mainly comes from the rituals and feeling of collective effervescence and must be seen as part of community rituals. Searching for the pharmacological high was neither the prime nor the most common explanation for using cocaine among the participants, but 
seemed to play an important part in the feeling of a state of collective effervescence derived from using cocaine.

\section{To Control, Extend and Intensify Drinking to Intoxication}

Participants explained that their most important motivation for using cocaine was to control, extend and intensify nighttime drinking. The control aspect was central to them because of their desire to "sober up" during escalating alcohol consumption, and to reduce hangover symptoms. Consequently, they perceived cocaine as a means of navigating their drinking to intoxication and therefore integral to their drinking experiences. These experiences were described as social in nature, and had a ritual character, adding to the rituals central in drinking together (Douglas, 2013). Participants explained that the rituals in cocaine use, such as snorting in a toilet in secrecy, intensified the feeling of pleasure in their clubbing.

Some young adults expressed concerns about being too drunk and insufficiently in control when partying. Cocaine solved the problem. With cocaine, they said they could drink as much as they wanted without getting drunk, in contrast to the commonly experienced alcohol intoxication when drinking without using cocaine. When asked how much he drank with and without cocaine, Eric replied,

Eric: I may drink eight beers and two drinks. And two shots. On a regular evening, maybe that is it, but when you take cocaine you have the feeling that you are sober all the time. You can drink as much as you want. I do not really know how much you can drink then, but it can be a $\operatorname{lot}[\ldots]$

Interviewer: Could it be twice as much?

Eric: Yes, it can. (Eric, 23-year-old male, frequent user)

Eric talked about drinking extensive amounts as nothing extraordinary; rather, it was something he did easily when using cocaine, and was part of the typical ritual of cocaine use. These young adults 
wanted to achieve the optimally balanced drunkenness level (described by them as a "buzz") and cocaine assisted them in that pursuit. They used cocaine to negotiate the border between chaos and order when clubbing. Dylan, a 31-year-old frequent user who claimed to have extensive knowledge of cocaine use, said: "You can be very steady, even if your alcohol concentration is over 1.5." He meant that his blood alcohol concentration (BAC) level was higher than 1.5\%, and the argument was that more injuries and damage might occur without cocaine use, because more people may be persistently and excessively drunk while using alcohol on its own. The ability to drink a lot and still continue partying was explained as valuable, as was the feeling of a controlled risk, regulated excess, or a controlled loss of control (Hayward, 2002; Measham, 2002). They maneuvered along a thin line - on the one hand seeking to lose control, while simultaneously retaining some degree of control anyway. Controlled loss of control can in this study be described as a combined feeling of intoxication and control, where alcohol played the role of the intoxicant, enabling them to break norms, and cocaine that of the control factor. They were able to engage in partying, dancing, flirting - all types of clubbing rituals - to a greater extent when they felt more sober, although intoxicated.

"You can drink as much as you want when using cocaine" was a common expression, as these young adults wanted to be able to keep the party going. Many appreciated the feeling of being more awake despite their intention of getting drunk. Terry noted how the issue of control, in a kind of calculated hedonism, was not related to user experience, as even first-time cocaine users would remain in control while drinking heavily. He said,

You do not lose control on cocaine. If it is the first time, and if you are drunk and mad, and take a key or a line, then you are awake and more balanced. (Terry, 30-year-old male, frequent user)

He said he felt sure about this point concerning control, even if it was the first time a person tried cocaine. The calculated hedonism occurred in the form of practices shared with others, and can be analyzed as interaction ritual chains, as they repeat the patterns of drinking and using cocaine together. In addition, the participants said that the issue of control was important to them, in order to be "more 
balanced", as Terry said. They preferred what can be interpreted as an extension of controlled loss of control, reflecting a double dimension with an element of time where cocaine was repeatedly used to facilitate a continuation of the party. The initial element was to use cocaine to augment alcohol intoxication. However, this was extended by new circles of additional cocaine use in order to regain control followed by prolonged drinking. This extension was perceived as a controlled loss of controlin control. They wanted a manageable "timeout" that lasted longer than a couple of hours, and cocaine made them feel in control again after drinking heavily, more vigilant and alert. They wanted to reach "the desired state of intoxication" (Measham, 2002, p.356). The desired state of controlled loss of control made them feel more "alive" and "in the know", by engaging in what they see as voluntary risk-taking behavior. This state of mind can also be called controlled euphoria. William explained how he felt attentive and alert, although he had had a lot to drink:

I feel that I get a lot more vigilant. I feel more fit, and much more on the alert. It [cocaine] also makes you feel less drunk. You just feel more bright and ready. You get a little bit steady. (William, 28-year-old male, frequent user)

This intensified clubbing, while still in control, is something the participants said they greatly appreciated, because they wanted the party to last longer. William and others wanted to be able to party throughout the night without falling asleep. In addition, many spoke about the reduced risk of violence. Liam explained:

If someone has had a lot to drink and start to talk rubbish and may do something crazy or get angry and break windows, then one may take cocaine and get a bit steadier, and then you can continue the conversation. (Liam, 28-year-old male, frequent user)

Several participants defended their use of cocaine because they believed that the risks of violence were lessened if they "sobered up" with the help of cocaine. With cocaine, they were able to lengthen the 
party without the more serious consequences they experienced from alcohol intoxication and using other substances, such as amphetamines, which they said gave them a hard "downer".

In addition to reduced comedown symptoms, many also emphasized using cocaine to reduce and control hangover symptoms from alcohol. Dylan put it like this:

If you want not to have to worry about the day after, cocaine is unfortunately very useful, and relatively available [...] That is why I have used it more often, because I can just sleep it off, and then it feels safe. (Dylan, 31-year-old male, frequent user)

When they used cocaine together with alcohol, participants felt they were less hung over the day after partying, even if they drank a lot. Many said they experienced milder hangovers if they drank in combination with cocaine, and this made cocaine an ideal substance. Dylan said "unfortunately" because he also talked about the long-term consequences and said he did not want to develop dependency or get into other trouble. For cocaine users, hangovers seemed to be least problematic when cocaine was used together with alcohol. Reducing hangover or comedown symptoms can be seen as a part of the control the cocaine users wanted to embrace when clubbing.

Study participants emphasized that cocaine use in clubs was combined with drinking, and the related rituals, to achieve a controlled loss of control. This was true for both the night out partying, and for the comedown the day after. They believed cocaine use made them capable of greater alcohol consumption without having to suffer the loss of control resulting from drunkenness or hangovers. In short, their cocaine use motivations appeared primarily connected to alcohol, especially to the aspects of controlling, managing, intensifying and extending drinking to intoxication.

Together, these three explanations illuminate cocaine rituals in a club culture context marked by alcohol intoxication; young adults used cocaine together with friends to increase the feeling of unity by shared transgression, visible in an alternative sense of a "high" through a feeling of collective effervescence. These results show how the culture surrounding cocaine use in clubs reflects more complex issues than a simple hunting for the high. 


\section{Discussion}

This study shows how cocaine rituals are important to recognize for a broader understanding of cocaine use in clubs. Cocaine use is analyzed as an extension of the culture of drinking to intoxication and, more specifically, as a way to manage, control and intensify partying. Drivers for cocaine use are largely connected to alcohol and the related rituals. Together this internal relationship between alcohol and cocaine highlights a club phenomenon in which young people use cocaine to strengthen the feeling of collective effervescence and excess.

Their repeated cocaine use was analyzed as a ritual connected to unity with friends and collective effervescence (Collins, 2004; Tutenges, 2013) that made going out more fun than drinking alone could, and hence strengthened the interaction rituals in the nightlife setting, by adding positive emotional energy and a kind of "electricity" that otherwise did not occur (Collins, 1993). The illegality contributed to the feeling of a "sneaky thrill" (Katz, 1988) that the young adults appreciated, and the characteristics of the alcohol party culture were expanded, modified and enhanced through the use of cocaine. Simultaneously, they describe using cocaine in order to drink in a more controlled way. The duality of control and intensifying shows that, at one moment, they wanted to feel under the influence, and, at the next, to exercise control as well. By analyzing the young adults' narratives as interaction rituals (Collins, 2004), this study illuminates more clearly how the culture around the use of cocaine is perceived as embedded in the culture of drinking to intoxication.

It is a paradox that young adults used cocaine (supposably more unconventional behavior) in order to regulate drinking (supposably more conventional behavior). They used a substance that is illegal and less socially accepted, to regulate a legal and more accepted activity, and break one norm so as not to break another, to them more important (although not illegal), norm. What seemed to be of importance to them was to not transcend the line from socially acceptable to unacceptable, and they did so by using an illegal and less accepted substance. By describing this paradox, this study complements existing research and adds insights by presenting an understanding of how the use of cocaine and related rituals is heavily intertwined in the culture of clubbing. The three explanations 
these young adults gave for their cocaine use were central to their perception of cocaine use in clubs and reveal insights into the logic of this use.

Several participants defended their use of cocaine because they believed that the risks of violence were lessened if they "sobered up" with the help of cocaine. They thought the ability to regain control when they found themselves drunk in the club was the drug's best property, and the combination of alcohol and cocaine was accordingly considered less inciteful of violence than drinking without cocaine. This line of reasoning can be interpreted as an intuitive strategy for reducing problems related to clubbing; these clubbers thought they were more in control and less vulnerable to the negative effects of alcohol when combining it with cocaine. Research on cocaine use and violence is mixed, as some studies suggest that the combination of alcohol and cocaine may increase violent tendencies (Pennings, Leccese, \& de Wolff, 2002) or may not necessarily do so (Lightowlers \& Sumnall, 2014). The empirical question of cocaine use and violence is also related to gender ${ }^{5}$ (Goldstein et al., 1991). It can be argued that these young adults perceived cocaine use as a strategy to reduce problems related to drinking, as demonstrated in the narratives about the controlled loss of control, alleviation of hangover symptoms, and the relative safety of cocaine use. This tactic can also be understood as a strategy for managing partying, because cocaine use was generally described in terms of offering multiple advantages for this activity.

According to Goffman (1990), social structures are created and recreated through interaction rituals. The links to nighttime drinking emerged naturally in the analysis of rituals surrounding cocaine use, where cocaine use seems to be an important part of the social encounters and rituals in the "club machinery". These encounters created emotional energy (Collins, 1993), a feeling of friendship, stronger social bonds and solidarity, as shown in other studies (Kavanaugh \& Anderson, 2008). Group solidarity and emotional energy in individuals are outcomes of interaction rituals (Collins, 2004, p.48), and this was highly visible in the participants' narratives on cocaine use. The cocaine rituals accompany the nighttime drinking rituals in multiple ways, ranging from snorting in clubs, to prolonging drinking to intoxication, to enhancing the feeling of fellowship with friends. Interaction rituals are important in drug use, as they are in drinking (Douglas, 2013; Järvinen, 2003). The sense of 
unity with fellow cocaine users was a central ritual that created boundaries of inclusion and solidarity (Collins, 2004; Kavanaugh \& Anderson, 2008); in that sense, it can easily be compared to the central elements in drinking together, although cocaine use was perceived to create a stronger and a more intense social element because of its illegality and transgression. For instance, when hiding in public places to snort cocaine, the young adults felt a community that is analyzed as a collective effervescence (Tutenges, 2013). The framework of interaction rituals chains has helped understand the explanations offered for using cocaine, by offering a framework to comprehend the calculated hedonism that drinking and use of drugs can be (Szmigin et al., 2008).

The social bonds were strengthened because of the rituals, and the feeling of a "sneaky thrill" was caused by the illicit nature of the use (Katz, 1988). The participants felt "pumped up" with emotional strength from the group interaction, because of this thrill (Collins, 2004, p.108). The use of keys for snorting was described as the single most important ritual, which made their use of cocaine feasible in clubs and allowed them to communicate about and engage in use without fear of being caught. This kind of secret language also made the social encounter of snorting more special to the users. Even if they sometimes went to the toilet to use alone, snorting still created a sense of community among those clubbing together, echoing Rothenbuhler's (1998) description of rituals as collectively structured performances that embody cultural codes, even when they are done individually. Even solo activity can be understood as part of interaction ritual chains because this activity is a result of earlier interaction that motivates it (Collins, 2004, p.6). The interaction rituals, carried out alone or together with peers, created a kind of fictive kinship that seemed very valuable to the participants.

Cocaine use - as described by these young adults - is interpreted as a strategy to engage in drinking in a more extreme way, to achieve excitement and an adrenalin kick. In Norwegian society, drinking to intoxication is not seen as an extreme behavior. In a subcultural understanding, the use of cocaine combined with alcohol can be a means to achieve the right degree of excess. Underpinning this study is therefore a perception of subculture not in a traditional descriptive sense (Gelder, 2005; Golub, Johnson, \& Dunlap, 2005; Hebdige, 1988), but rather of the broader club culture where young 
people participate in the specific subcultural behaviors with only some aspects of their identities. They do not go "all in", like subcultural identities often demand. This also resonates with the findings in a Danish club study, where the cocaine users perceived themselves as "normal youth", and not part of a subculture (Demant, 2010, p.160). Participants in this study felt they lived ordinary lives and appeared to use cocaine primarily to augment their mainstream parties, and not to engage in or identify with a distinct, perhaps more socially deviant, cocaine subculture. They did not seem to be marginalized or severely affected by their cocaine use, as most of them were working or studying. This sociocultural perspective enables a deeper understanding of these phenomena and makes meaning and motives for cocaine use more visible. While there may be conflicts between risky leisure roles and the mainstream professional ones, this did not appear to be the case for most of these users. For them, cocaine use seemed to be integrated into drinking, in line with social norms of what is socially acceptable in a party-culture that was seen as mainstream more than subcultural, and which could be combined with their obligations. They managed their professional identities despite partying hard, and their use was at the right point of coolness: illegal, but not perceived as too risky. These findings show how different drugs, with different pharmacological effects and different social meanings, are used to achieve complex social goals, determined by specific cultural contexts, like drinking norms, identity constructions, regulatory contexts, and user groups.

Based on the findings, it can be asked whether the use of cocaine simply supplements the culture of drinking to intoxication, or if it constitutes a distinct culture. Cocaine use has often been understood primarily in terms of the high (Weiss et al., 2002), and descriptions in popular culture may have contributed to this dominant, yet overly simplistic, perception. This study has unveiled nuances of cocaine use in a nightlife setting based on a focus on the specific social context of and rituals around the use and has shown how cocaine helps navigate the fine line between being "wasted" and being in control, although intoxicated. Judging by the group examined for this study, attention on the "high" seems to be heavily influenced by popular culture, such as films and music, and not rooted in users' own narratives. Cocaine provided the possibility for a controlled loss of control (Hayward, 2002; Measham, 2002), and in addition a controlled loss of control - in control, as an improvised strategy for the users to reduce their perceived problems related to intoxication. This additional time 
aspect has not previously been acknowledged. The concept of "controlled loss of control" has here been expanded to encompass how the participants use cocaine to regain control after extended partying. These narratives echo and broaden the results from an empirical Italian study where clubbers wanted to find the "right level of intoxication" (Aresi \& Pedersen, 2016), and it appears that young adults in clubs in Norway use cocaine to reach this level. The cultural context was provided by drinking to intoxication, and for these young adults, cocaine fits well into the familiar cultural script. These findings can teach us something important about young people in contemporary society and the need to be in control, and within this control aspect, health seems to be an important factor (Wiklund, Jonsson, Coe, \& Wiklund, 2019). Participants employed cocaine as a kind of band-aid or first aid kit to regulate their night out, and control seemed to be of great significance to them.

Some study limitations should be acknowledged. First, while the results provide insights into drug use in the Oslo club scene, they cannot necessarily be generalized to other cities, because other countries and cities have different patterns of cocaine and alcohol use. Second, not all explanations were equally important to all participants, although most of them emerged frequently and were emphasized by most participants. Despite these limitations, the insights from this study may have implications for practice, specifically prevention measures, and can be used to tailor more specific prevention messaging and to develop campaigns for minimizing drug use risks. As earlier studies have requested, it is crucial to find ways to engage this key population of active users of club drugs at "individual, subcultural, and structural levels in order to more fully support health promotion among young adults" (Kelly, Parsons, \& Wells, 2006, p.893). As shown in the results, the cocaine-induced perception of being sober can provide the sense of control and lead to additional consumption of alcohol in even larger quantities, which may have severe consequences. Understanding such particular hazards of combined alcohol and cocaine use may thus be of importance. These insights may contribute to the argument for making relevant information in clubs more available, which should be customized to the local use pattern, as these may vary considerably. 


\section{Conclusion}

Participants used cocaine in club settings to control and extend their drinking to intoxication; such modes of cocaine use are consequently interpreted as a way to manage and intensify their nightlife drinking rituals in a collective effervescence. These findings improve our understanding of cocaine use by seeing it as part of a nightlife setting because this context seems to shape the drug-use behaviors. The rituals central to cocaine use accompany the rituals central in drinking. To the young adults, cocaine use seems primarily to contribute extra rituals to the perfect party, by expanding the drinking experience. They see cocaine both as a medium of controlled drinking to intoxication, and of partying harder. Considering the interlinked relationship between cocaine use and drinking adds to our knowledge base of drinking, clubbing, and cocaine use and its related rituals, and can inform policy strategies for both cocaine and alcohol prevention. Future studies should focus on subjective explanations for use and on probing the associated cultures. This study has highlighted how cocaine use and its related rituals are heavily intertwined in the local drinking culture, and how cocaine use in clubs may reflect more complex issues than a simple search for the high.

\section{Acknowledgements}

I would like to thank the young adults who talked about their club drug experiences, and the research assistants who helped with recruitment. In addition, I would like to thank Sveinung Sandberg, Willy Pedersen, Janne Scheffels, Kristin Buvik and several colleagues at the Norwegian Institute of Public Health for providing valuable comments to the manuscript, and the Editor and anonymous reviewers whose suggestions improved the article. 


\section{Declaration of Conflicting Interests}

The author declared no potential conflicts of interest with respect to the research, authorship, and/or publication of this article.

\section{Funding}

This research is funded by the Norwegian Institute of Public Health, and did not receive any specific grant from funding agencies in the public, commercial, or not-for-profit sectors.

\section{Notes}

1. Concurrent use of cocaine and alcohol also has been associated with greater risk of sudden death than use of cocaine alone (Andrews, 1997).

2. The reason for interviewing twice was primarily to check findings from the data material.

3. Seven of those who were contacted, were not interviewed. Only two out of these said they had changed their mind and did not want to be interviewed; the remaining five were either out of town or did not have time to be interviewed when asked.

4. The remaining interviews were conducted in the participant's home (1), outdoors (3), and at a shopping mall restaurant (1). When the interview was conducted in the office, no-one else was present in the room beside the participant and the researcher.

5. For instance, one particular study shows that violence for males more often occurs in the course of economic crimes, while for females, violence most often occurs in non-drug-related disputes (Goldstein, Bellucci, Spunt, \& Miller, 1991, p.365). 


\section{References}

Andrews, P. (1997). Cocaethylene toxicity. Journal of addictive diseases, 16(3), 75-84.

Aresi, G., \& Pedersen, E. R. (2016). 'That right level of intoxication': A Grounded Theory study on young adults' drinking in nightlife settings. Journal of Youth Studies, 19(2), 204-220.

Babor, T. F., Caetano, R., Casswell, S., Edwards, G., Giesbrecht, N., Graham, K., . . Rossow, I. (2003). Alcohol: no ordinary commodity: research and public policy. New York: Oxford University Press.

Berridge, V., Herring, R., \& Thom, B. (2009). Binge drinking: a confused concept and its contemporary history. Social history of medicine, 22(3), 597-607.

Collins, R. (1993). Emotional energy as the common denominator of rational action. Rationality and society, 5(2), 203-230.

Collins, R. (2004). Interaction ritual chains. New Jersey: Princeton University Press.

Dackis, C. A., \& O'Brien, C. P. (2001). Cocaine dependence: a disease of the brain's reward centers. Journal of substance abuse treatment, 21(3), 111-117.

Decorte, T. (2001). Drug users' perceptions of 'controlled'and 'uncontrolled'use. International Journal of Drug Policy, 12(4), 297-320.

Decorte, T., \& Slock, S. (2005). The taming of Cocaine II. Brussels: VUB Brussels University Press.

Demant, J. (2010). Kokain og alkohol - en kontrollabel cocktail? In: M. Järvinen, Demant, J., and $\emptyset$ stergaard, J. (Ed), Stoffer og natteliv (p. 146-163). Copenhagen: Hans Reitzels Forlag.

Demant, J., \& Ravn, S. (2010). Identifying drug risk perceptions in Danish youths: Ranking exercises in focus groups. Drugs: Education, Prevention and Policy, 17(5), 528-543.

Demant, J., Ravn, S., \& Kaae, A. M. (2011). The glamour makes the difference. Discourses on cocaine, ecstasy and amphetamine in Danish media. In: J. Fountain, Asmussen Frank, V., Korf D.J. (Ed), Messages, Markets and Methods. Dynamics in European Drug Research (p. 136-151). EU: Pabst Science Publishers.

Demant, J., Ravn, S., \& Thorsen, S. K. (2010). Club studies: methodological perspectives for researching drug use in a central youth social space. Leisure Studies, 29(3), 241-252.

Douglas, M. (2013). Constructive drinking. (Vol. 10). London: Routledge.

Durkheim, E. (1965 [1915]). The Elementary Forms of Religious Life. New York: The Free Press.

Edland-Gryt, M., Sandberg, S., \& Pedersen, W. (2017). From ecstasy to MDMA: Recreational drug use, symbolic boundaries, and drug trends. International Journal of Drug Policy, 50, 1-8.

EMCDDA. (2019). European Drug Report 2019. Lisbon: EMCDDA. EMCDDA.

Esteve-Arenys, A., Gracia-Rubio, I., Cantacorps, L., Pozo, O. J., Marcos, J., Rodríguez-Árias, M., . . . Valverde, O. (2017). Binge ethanol drinking during adolescence modifies cocaine responses in mice. Journal of Psychopharmacology, 31(1), 86-95.

Fagan, J. (1994). Women and drugs revisited: Female participation in the cocaine economy. Journal of drug issues, 24(2), 179-225.

Gawin, F. H. (1991). Cocaine addiction: psychology and neurophysiology. Science, 251(5001), 15801586.

Gelder, K. (2005). The subcultures reader. New York: Routledge.

Gmel, G., Rehm, J., \& Kuntsche, E. (2003). Binge drinking in Europe: definitions, epidemiology, and consequences. Sucht, 49(2), 105-116.

Goffman, E. (1990). The Presentation of Self in Everyday Life. London: Penguin Books.

Goffman, E. (2008 [1967]). Interaction ritual: essays on face-to-face interaction. New Brunswick: AldineTransaction.

Goldstein, P. J., Bellucci, P. A., Spunt, B. J., \& Miller, T. (1991). Volume of cocaine use and violence: A comparison between men and women. Journal of drug issues, 21(2), 345-367.

Golub, A., Johnson, B. D., \& Dunlap, E. (2005). Subcultural evolution and illicit drug use. Addiction Research \& Theory, 13(3), 217-229.

Grinspoon, L., \& Bakalar, J. B. (1985). Cocaine: A drug and its social evolution. New York: Basic Books. 
Harris, D. S., Everhart, E. T., Mendelson, J., \& Jones, R. T. (2003). The pharmacology of cocaethylene in humans following cocaine and ethanol administration. Drug and alcohol dependence, 72(2), 169-182.

Hayward, K. (2002). The vilification and pleasures of youthful transgression. In: J. Muncie, Hughes, G. and McLaughlin, E. (Ed), Youth Justice: Critical Readings (p. 80-94). London: Sage.

Hebdige, D. (1988). Subculture: The meaning of style. New York: Routledge.

Heradstveit, O., Skogen, J. C., Edland-Gryt, M., Hesse, M., Vallentin-Holbech, L., Lønning, K. J., \& Sivertsen, B. (2020). Self-reported illicit drug use among Norwegian university and college students. Associations with age, gender and geography. Frontiers in Psychiatry, 11, 1424.

Holstein, J. A., \& Gubrium, J. F. (2004). The active interview. In: D. Silverman (Ed), Qualitative research: Theory, method and practice (Vol. 2, p. 140-161). London: SAGE.

Hsieh, H.-F., \& Shannon, S. E. (2005). Three approaches to qualitative content analysis. Qualitative health research, 15(9), 1277-1288.

Jones, A. W. (2019). Forensic drug profile: cocaethylene. Journal of analytical toxicology, 43(3), 155160.

Järvinen, M. (2003). Drinking rituals and drinking problems in a wet culture. Addiction Research \& Theory, 11(4), 217-233.

Järvinen, M., \& Ravn, S. (2011). From recreational to regular drug use: qualitative interviews with young clubbers. Sociology of health \& illness, 33(4), 554-569.

Katz, J. (1988). Seductions of Crime: Moral and Sensual Attractions in Doing Evil. New York: Basic Books.

Kavanaugh, P. R., \& Anderson, T. L. (2008). Solidarity and drug use in the electronic dance music scene. The Sociological Quarterly, 49(1), 181-208.

Kelly, B. C., Parsons, J. T., \& Wells, B. E. (2006). Prevalence and predictors of club drug use among club-going young adults in New York City. Journal of Urban Health, 83(5), 884.

Kuntsche, E., Rehm, J., \& Gmel, G. (2004). Characteristics of binge drinkers in Europe. Social science \& medicine, 59(1), 113-127.

Kuypers, K., Steenbergen, L., Theunissen, E., Toennes, S., \& Ramaekers, J. (2015). Emotion recognition during cocaine intoxication. European Neuropsychopharmacology, 25(11), 19141921.

Kvale, S. B., Svend (2009). Interviews. Learning the craft of qualitative research interviewing. Thousands Oak: SAGE Publications.

Lightowlers, C., \& Sumnall, H. (2014). A violent mix? The association between concurrent alcohol and cocaine use and violence amongst young people in England and Wales. Drugs: Education, Prevention and Policy, 21(2), 131-139.

Martin, G., Macdonald, S., Pakula, B., \& Roth, E. A. (2014). A comparison of motivations for use among users of crack cocaine and cocaine powder in a sample of simultaneous cocaine and alcohol users. Addictive Behaviors, 39(3), 699-702.

Measham, F. (2002). "Doing gender" - "doing drugs": Conceptualizing the gendering of drugs cultures. Contemporary Drug Problems, 29(2), 335-373.

Measham, F., Aldridge, J., \& Parker, H. (2001). Dancing on Drugs: Risk, health and hedonism in the British club scene. London: Free Association Books.

Measham, F., \& Brain, K. (2005). 'Binge'drinking, British alcohol policy and the new culture of intoxication. Crime, media, culture, 1(3), 262-283.

Moore, D. (2010). Beyond disorder, danger, incompetence and ignorance: Rethinking the youthful subject of alcohol and other drug policy. Contemporary Drug Problems, 37(3), 475-498.

Mäkelä, P., Fonager, K., Hibell, B., Nordlund, S., Sabroe, S., \& Simpura, J. (2001). Episodic heavy drinking in four Nordic countries: a comparative survey. Addiction, 96(11), 1575-1588.

Nordfjærn, T., Bretteville-Jensen, A. L., Edland-Gryt, M., \& Gripenberg, J. (2016). Risky substance use among young adults in the nightlife arena: An underused setting for risk-reducing interventions? Scandinavian Journal of Public Health, 44(7), 638-645. 
Nordfjærn, T., Edland-Gryt, M., Bretteville-Jensen, A. L., Buvik, K., \& Gripenberg, J. (2016). Recreational drug use in the Oslo nightlife setting: Study protocol for a cross-sectional time series using biological markers, self-reported and qualitative data. BMJ open, 6(4).

Nutt, D. J., King, L. A., \& Phillips, L. D. (2010). Drug harms in the UK: a multicriteria decision analysis. The Lancet, 376(9752), 1558-1565.

Palamar, J. J., Davies, S., Ompad, D. C., Cleland, C. M., \& Weitzman, M. (2015). Powder cocaine and crack use in the United States: An examination of risk for arrest and socioeconomic disparities in use. Drug and alcohol dependence, 149, 108-116.

Pennings, E. J., Leccese, A. P., \& de Wolff, F. A. (2002). Effects of concurrent use of alcohol and cocaine. Addiction, 97(7), 773-783.

Ravn, S. (2012). Managing drug use in Danish club settings: A normalized enterprise? Young, 20(3), 257-276.

Rolando, S., Törrönen, J., \& Beccaria, F. (2014). Boundaries between adult and youth drinking as expressed by young people in Italy and Finland. Young, 22(3), 227-252.

Rothenbuhler, E. W. (1998). Ritual communication: From everyday conversation to mediated ceremony. California: SAGE Publications.

Silverman, D. (2009). Doing Qualitative Research. (Vol. Third edition). London: Sage Publications L.td.

Szmigin, I., Griffin, C., Mistral, W., Bengry-Howell, A., Weale, L., \& Hackley, C. (2008). Re-framing 'binge drinking' as calculated hedonism: Empirical evidence from the UK. International Journal of Drug Policy, 19(5), 359-366.

Tutenges, S. (2013). Stirring up effervescence: An ethnographic study of youth at a nightlife resort. Leisure Studies, 32(3), 233-248.

Uosukainen, H., Tacke, U., \& Winstock, A. R. (2015). Self-reported prevalence of dependence of MDMA compared to cocaine, mephedrone and ketamine among a sample of recreational poly-drug users. International Journal of Drug Policy, 26(1), 78-83.

van der Poel, A., Rodenburg, G., Dijkstra, M., Stoele, M., \& van de Mheen, D. (2009). Trends, motivations and settings of recreational cocaine use by adolescents and young adults in the Netherlands. International Journal of Drug Policy, 20(2), 143-151.

Waldorf, D., Reinarman, C., \& Murphy, S. (1992). Cocaine changes: The experience of using and quitting. (Vol. 49). Philadelphia: Temple University Press.

Watters, J. K., \& Biernacki, P. (1989). Targeted sampling: options for the study of hidden populations. Social problems, 36(4), 416-430.

Weiss, R. D., Mirin, S. M., \& Bartel, R. L. (2002). Cocaine. Washington, DC: American Psychiatric Pub.

Wiklund, E., Jonsson, E., Coe, A.-B., \& Wiklund, M. (2019). 'Strong is the new skinny': navigating fitness hype among teenagers in northern Sweden. Sport, Education and Society, 24(5), 441454.

Williams, T. (1989). The cocaine kids. New York: Addison-Wesley Publishing Company, Inc.

Zinberg, N. E. (1984). Drug, Set and Setting. The Basis for Controlled Intoxicant Use. New York: Yale University Press.

Zyoud, S.H., Waring, W.S., Al-Jabi, S. W., \& Sweileh, W.M. (2017). Global cocaine intoxication research trends during 1975-2015: A bibliometric analysis of Web of Science publications. Substance Abuse Treatment, Prevention, and Policy, 12(1), 6-6. 


\section{Author Biography}

Marit Edland-Gryt is a Ph.D. candidate in Sociology at the University of Oslo and a researcher at the Norwegian Institute of Public Health. Her research is focused on sociological studies of young people and drug use. 